\title{
germectomies transalvéolaires des premières prémolaires
}

RÉSUMÉ Après avoir parcouru les différentes techniques de germectomie de prémolaires classiquement décrites, les auteurs présentent un protocole dont l'originalité consiste en une voie d'abord exclusivement transalvéolaire. Les avantages et les inconvénients per, post-opératoires de ces différentes techniques sont abordés.

\section{Gilbert POIROT}

Docteur en Sciences Odontologiques,

Ancien Chef de travaux

à la faculté de chirurgie dentaire

de Reims,

38 bis, rue de Courlany,

51100 Reims.

\section{Thierry GUÉRIN}

Ancien assistant à la faculté

de chirurgie dentaire

de Paris V,

17, rue des Petits Hôtels,

75010 Paris.

\section{MOTS CLÉS}

germectomie

prémolaire

techniques opératoires

suites opératoires 


\section{introduction}

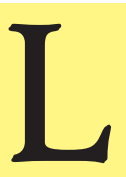

'indication des germectomies des prémolaires est essentiellement orthodontique, et il ne sera pas de notre propos d'en discuter les indications. L'orthodontiste évalue l'importance de la dysharmonie dento-maxillaire, le potentiel de croissance du sujet. Les indications se trouvent donc posées grâce à l'examen clinique et aux analyses céphalométriques[3, 11, 12, 14, 16].

Que doit-on attendre de l'acte chirurgical ? Quelle est l'influence de cette intervention sur le plan de l'évolution spontanée des canines, ainsi que celle sur le plan de la cicatrisation parodontale (gingivale et osseuse) ? Voici les questions auxquelles nous sommes confrontés.

La plupart du temps, les techniques classiques de germectomie présentent une voie d'abord vestibulaire. Une fois la contention de fin de traitement ortho- dontique retirée, on constate fréquemment la réouverture de l'espace $3 / 5$, ainsi qu'une bride fibreuse cicatricielle verticale le long du rempart alvéolaire.

Selon Prichard $34 \%$ des cas traités avec extraction des prémolaires présentent des réouvertures d'espace interdentaire ou des points de contacts insuffisamment serrés[15].

Deplagne constate que si la canine évolue en bonne place après germectomie de la première prémolaire, elle est en rotation de 30 à $40^{\circ}$ : moins de malposition mais plus de rotation[5].

La technique chirurgicale que nous proposons impose la présence de la molaire temporaire, et minimise les problèmes précédemment cités.

La technique procède par extraction de la molaire temporaire avec ensuite fractionnement du germe sans toucher la paroi osseuse alvéolaire qui l'entoure.

\section{les objectifs de la chirurgie de la germectomie de la première prémolaire}

Dans le cadre du traitement orthodontique, nous fixons les objectifs chirurgicaux auxquels ces germectomies doivent répondre :

- donner un traumatisme psychologique pré, per et post-opératoire minimal pour l'enfant pour lequel il s'agit le plus souvent de la première intervention chirurgicale ;

- se placer le plus près possible des conditions de la chute de la dent temporaire ;
- préserver le capital osseux, tissu au sein duquel la canine va évoluer ;

- éviter le pincement cicatriciel vestibulo-lingual de la table osseuse vestibulaire ;

- être peu traumatisant pour le parodonte et économe de tissus gingival et osseux, c'est-à-dire éviter les incisions de décharge, la mise au jour de l'os alvéolaire, les résections de table alvéolaire ; 
- avoir des suites opératoires (œdème, - être sans risque pour les structures douleur) les plus simples possibles ; nobles (nerf alvéolo-mandibulaire, nerf - ne pas léser les germes et les dents mentonnier). environnantes ;

\section{contre-indications et indications chirurgicales}

Une fois l'indication orthodontique posée, le chirurgien se doit d'intervenir le plus tôt possible. Il n'est pas nécessaire d'alourdir un traitement orthodontique qui est déjà suffisamment contraignant pour l'enfant.

- les contre-indications générales

Les contre-indications d'ordre général sont celles de toute chirurgie.

- les contre-indications locales

Les critères locaux parodontaux peuvent retarder l'intervention jusqu'à la maîtrise de l'inflammation. Cependant, chez des jeunes enfants, le contrôle total de l'inflammation sera difficile à obtenir[1, 9, 13, 19].

\section{- I'indication chirurgicale}

L'indication chirurgicale est posée devant le patient lors de la première consultation.

L'examen clinique est complété par la lecture de l'orthopantomogramme.

Le germe étant situé au-dessus de la dent permanente, il est rare de demander un bilan radiologique d'extention.

\section{la première consultation}

Le moment de la première consultation est primordial.

Le patient et les parents doivent être avertis par l'orthodontiste de la nécessité de cette première consultation qui permet :

- de faire connaissance avec un nouveau praticien ;

- d'expliquer simplement l'intervention : où, comment, qui sera dans la salle d'intervention. Nous devons informer le jeune patient, et répondre à ses interrogations ;

- de donner les conseils pré et post-chirurgicaux ;

- de prescrire une prémédication : sédative, antalgique, et si nécessaire antibiotique, et éventuellement un bilan d'hémostase ;

- d'obtenir le consentement éclairé du représentant légal. 


\section{techniques classiquement décrites}

Deux grandes classes existent : les interventions avec une voie d'abord vestibulaire, et celles avec une voie d'abord alvéolaire.

\section{- les voies d'abord vestibulaire}

Il existe un certain nombre de variantes de la voie d'abord vestibulaire selon les auteurs[2, 4-8, 17].

Ces techniques se déroulent en trois temps majeurs :

1 - réalisation d'un lambeau de pleine épaisseur, avec ou sans incision de décharge mettant à nu l'os alvéolaire ;

2 - trépanation osseuse vestibulaire plus ou moins importante, donnant accès au germe ;

3 - morcellement ou non du germe et exérèse par voie vestibulaire.
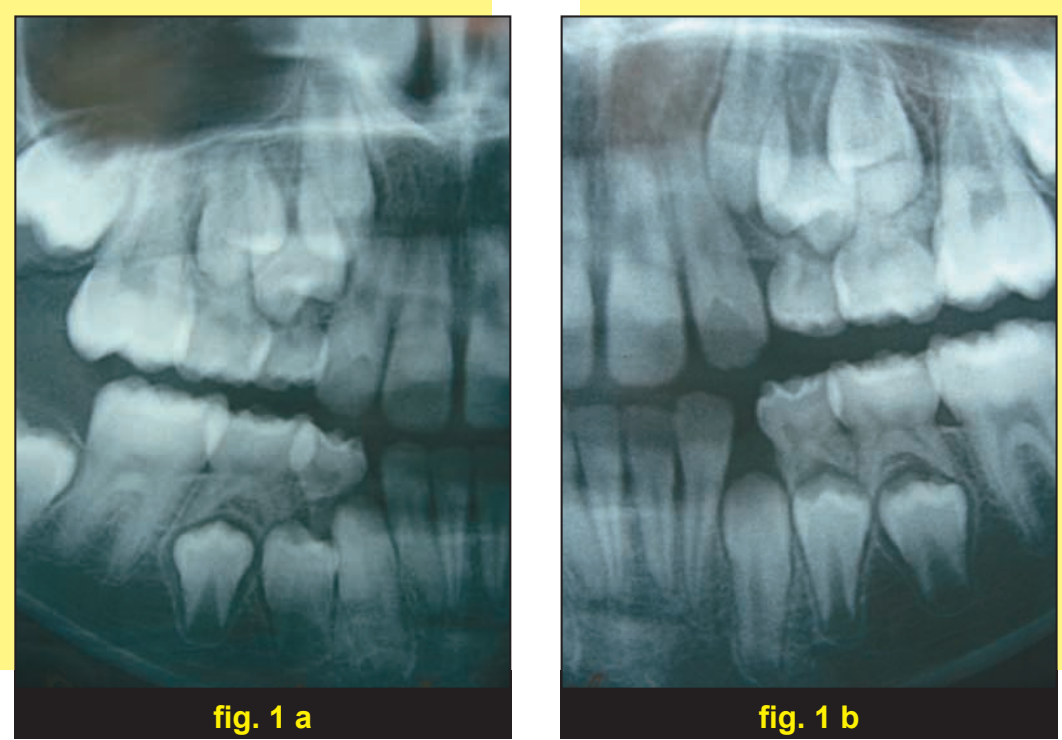

fig. 1 a et b Bilan radiologique préopératoire. Détails de la panoramique à droite (fig. 1 a) et à gauche (fig. 1 b). 
duelle étant la plupart du temps source d'une réouverture d'espace entre 3 et 5 (fig. 1 a et b) ;

- le risque de lésion nerveuse du nerf mentonnier au foramen homonyme ou en intra-osseux du nerf alvéolomandibulaire inférieur ;

- les projections des germes maxillaires dans le sinus lors d'élévations intempestives.

\section{la technique transalvéolaire}

La situation du germe de la prémolaire par rapport à celle de la dent temporaire est spécifique. En effet, les cryptes osseuses des prémolaires se développent entre les racines des molaires temporaires ; les canaux gubernaculaires

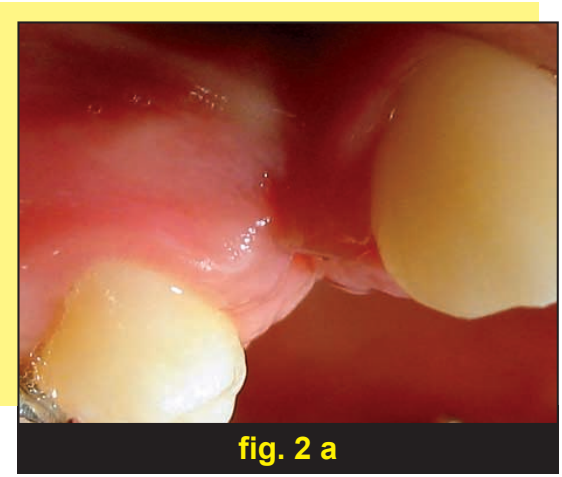

s'ouvrent le plus souvent au sommet du septum interradiculaire de la dent temporaire susjacente[10, 18] (fig. 2 a et b). Partant de cette situation anatomique, nous proposons une technique strictement transalvéolaire.

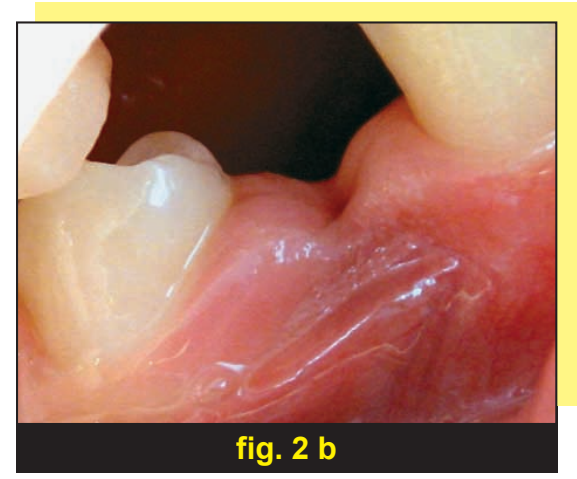

fig. 2 a et b Brides fibreuses après germectomie de 14 (fig. 2 a) et de 44 (fig. 2 b).

synopsis de l'intervention

(fig. 3,

fig. 4 a à $f$,

fig. 5 a à $d$ et fig. 6)

L'installation du patient, la désinfection de la zone à infiltrer puis à opérer, la pose des champs opératoires, le choix de la technique et de la molécule anesthésique, bien qu'étant incontournables sont considérés ici comme réalisés[9].

1 - Syndesmotomie et extraction de la dent temporaire.

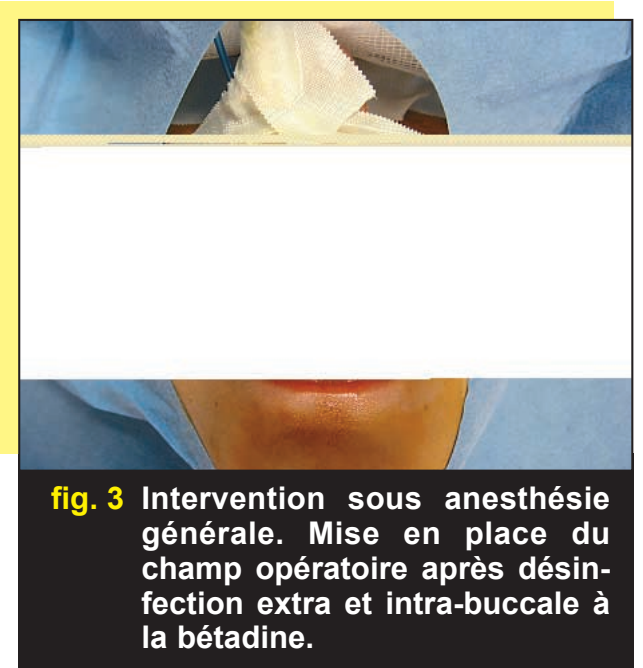



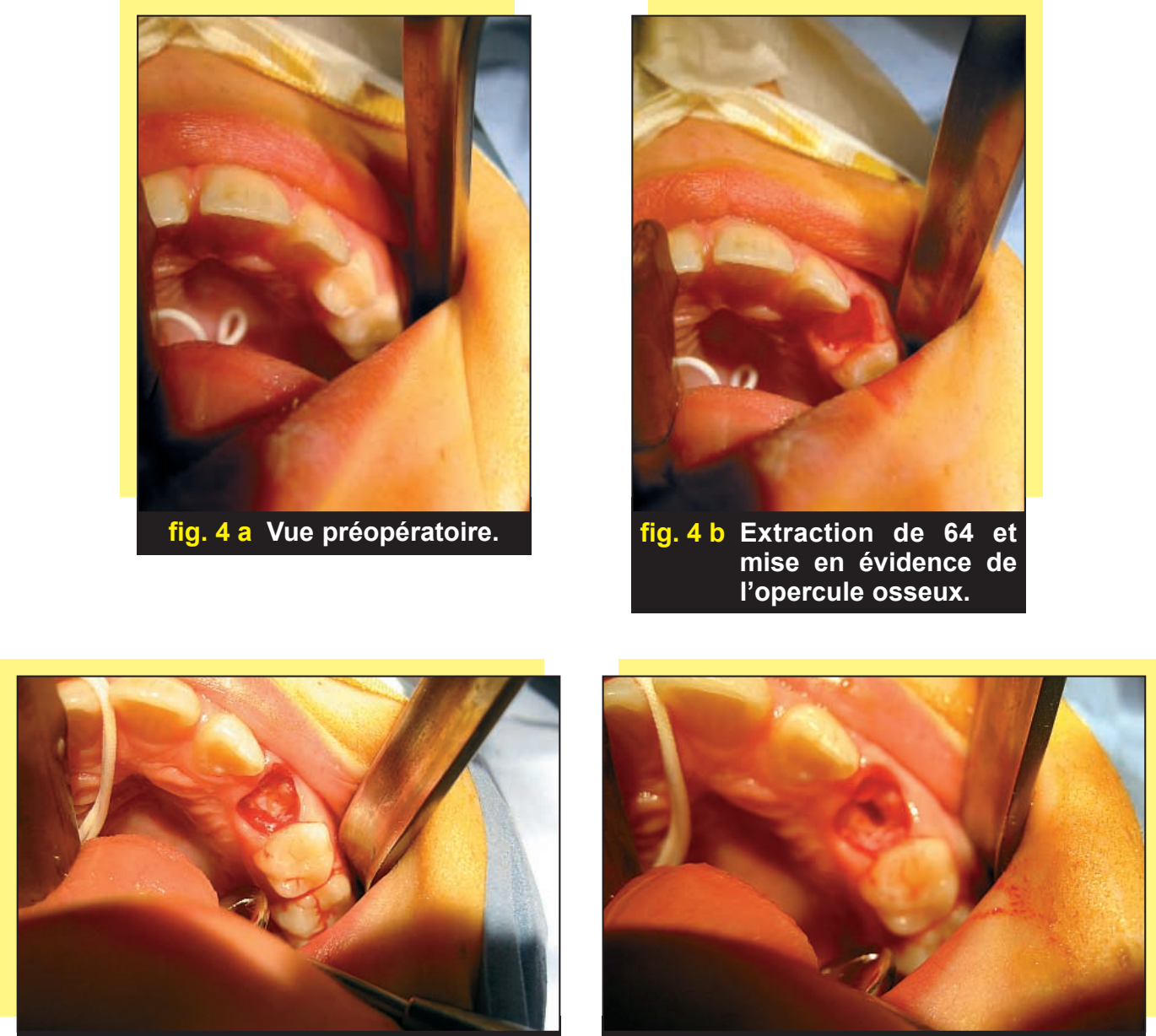

fig. 4 c Dégagement de l'opercule osseux et mise à jour de la face occlusale du germe de 24.

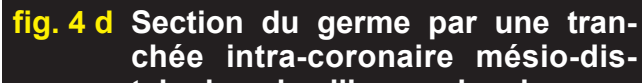
tale dans le sillon occlusal.
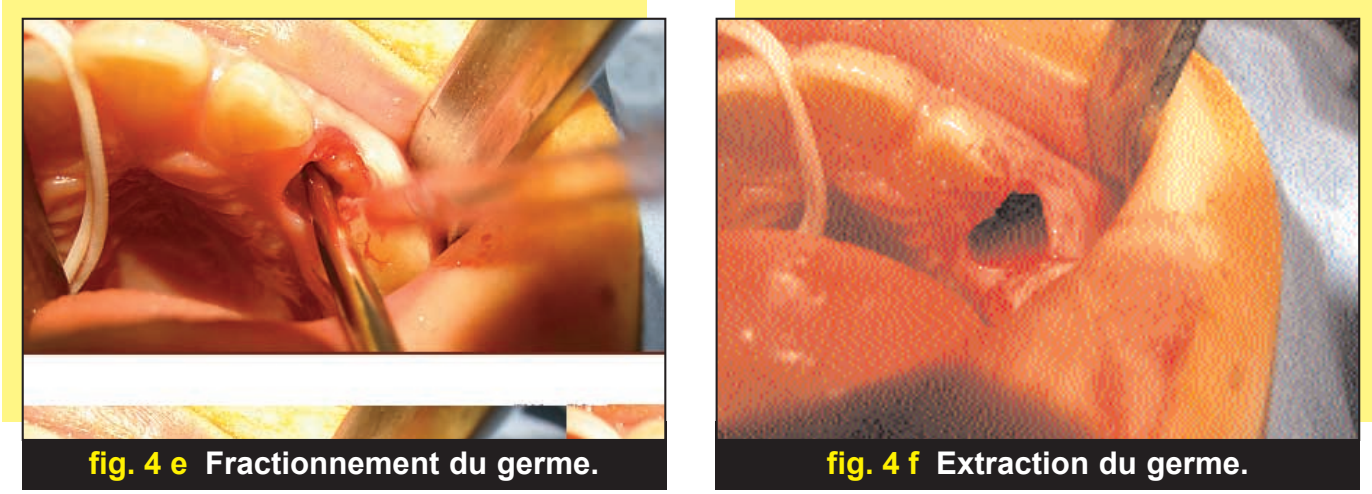

fig. 4 a à $f$ Germectomie de 24 . 

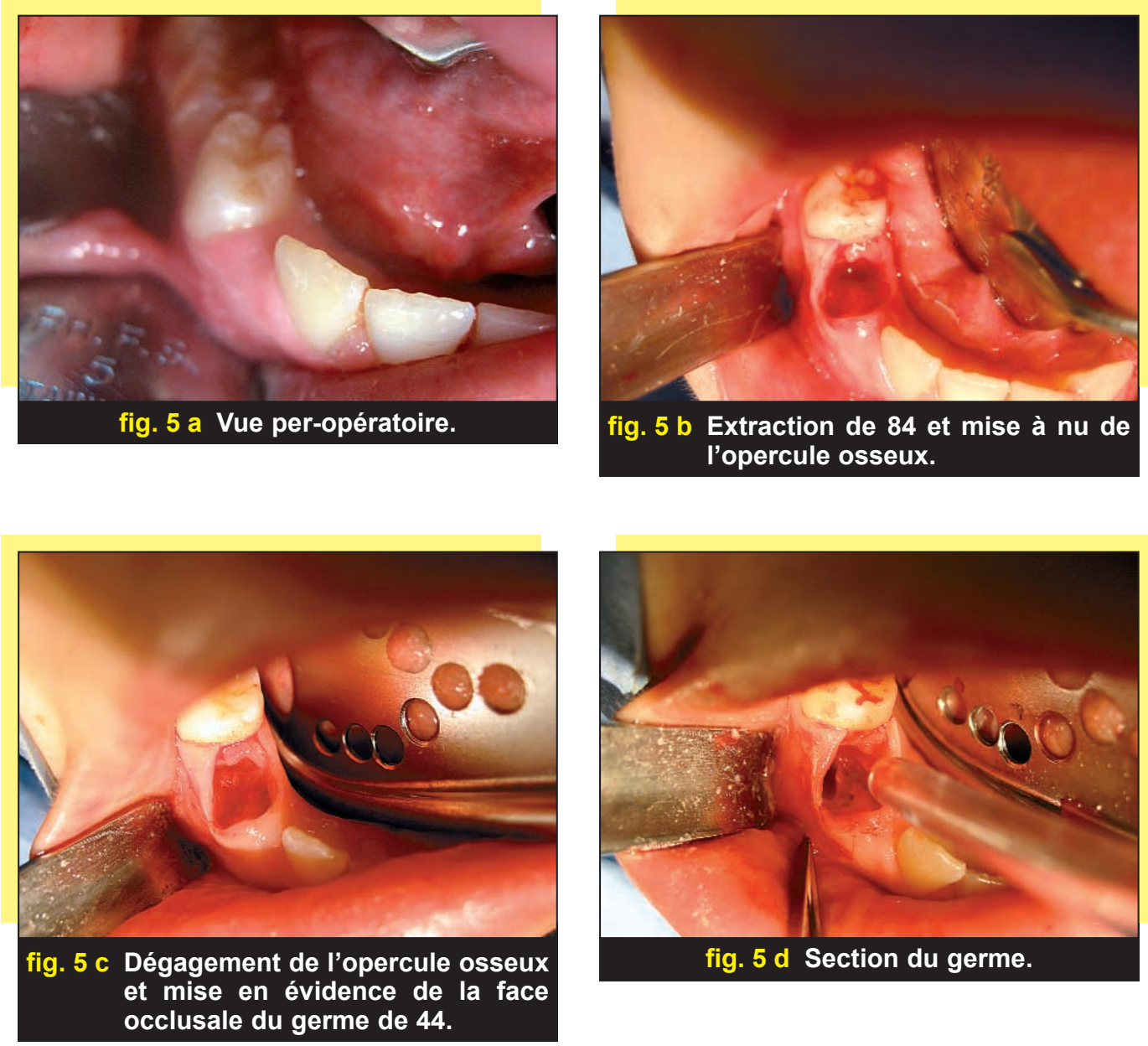

et mise en évidence de la face

fig. 5 d Section du germe.

\section{fig. 5 a à d Germectomie de 44.}

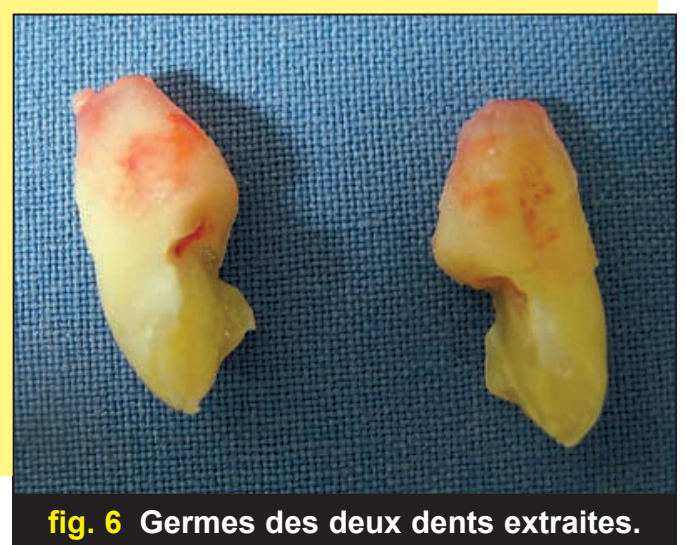


2 - Effondrement à la fraise tungstène, de l'opercule osseux séparant l'alvéole de la dent temporaire du sac péricoronaire du germe.

3 - Exérèse de la partie occlusale du sac et mise à nu de la partie occlusale du germe.

4 - Repérage anatomique du germe et détermination :

- du sillon mésio-distal occlusal ;

- de l'axe de la dent.

5 - Section du germe : réalisation d'une tranchée mésio-distale :

- épargnant les faces proximales du germe ;

- d'une largeur compensant le bombé vestibulaire de la dent.

Le siège de la tranchée mésio-distale est différent au maxillaire et à la mandibule :

- au maxillaire, elle répond au sillon occlusal mésio-distal de la dent ;

- à la mandibule, elle est à l'aplomb de la pointe cuspidienne vestibulaire.

Dans des cas de germe volumeux il est possible de compléter cette tranchée par une section vestibulo-linguale.

6 - Éclatement du germe.

7 - Exérèse des fragments de germe.

8 - Exérèse du sac folliculaire.

9 - L'hémostase est réalisée par compression.
- avantages de la technique

Les avantages de cette technique sont nombreux :

- absence de toute lésion parodontale ;

- suites opératoires presque nulles s'apparentant à celles endurées pour l'extraction d'une dent temporaire isolée ;

- saignement per- et post-opératoire nul ;

- pas de risque d'apparition de bride fibreuse cicatricielle et de réouverture d'espace ;

- absence de tendance à la rotation de la canine ;

- absence de risque de projection de germe dans le sinus pour les germes maxillaires ou de lésion du nerf alvéolaire inférieur et de ses branches (nerf mentonnier et nerf incisif) pour les germes mandibulaires.

- inconvénients de la technique

- Parfois, difficulté d'appréciation de l'axe des germes.

- Technique un peu plus longue pour les germes profonds.

- Position inconfortable de l'enfant: tête en hyperextention pour les germes maxillaires et en flexion pour les germes mandibulaires.

\section{conclusion}

La technique que nous proposons répond aux critères qui ont été fixés au préalable: préservation des tissus de soutien de la canine, respect des tables osseuses, suites opératoires a minima. C'est pour ces raisons que nous la préférons aux techniques classiquement décrites. 


\section{bibliographie}

1. Becker W, Berg L, Becker BE.

Evaluation à long terme du traitement parodontal et de sa maintenance chez 95 patients. Int J Parodont Dent Rest 1976;11:182-188.

2. Bernard JC, Boniver A. Technique originale de germectomie de première prémolaire définitive. Rev Belge Med Dent 1986;41(1):19-22.

3. Dahan MJ

La germectomie de la deuxième prémolaire. Etude gnathométrique longitudinale pour en déterminer l'indication. 1973;44(1):517-527.

4. Della Guardia LD, Erard J. La germectomie prémolaire inférieure. Rev Odonto Stomatol 1974;3(5):387-396.

5. Deplagne $\mathrm{H}$. Moyen d'éviter la rotation des canines après germectomie des premières prémolaires. Rev Orthop Dento Faciale 1985;19:591-593.

6. Duprat M.

Apport de la chirurgie : germectomie précoce et raisonnée de la première prémolaire. Rev Orthop Dento Faciale 1990;24:175-188.
7. Duclos P.

Problèmes chirurgicaux propres aux germectomies. Chir Dent France 1980;50(87):55-64.

8. Fave $A$, Hirigoyen $Y$, Vilain M.

Extractions dentaires : canines et autres dents incluses.

Encycl Med Chir (Elsiever, Paris), Stomatologie, 22096 A-10-2, 1989, 8p.

9. Guérin Th, Maman L, Wierzba CB.

Anesthésie loco-régionale :

II. Le choix des solutions et des molécules. Actual Odontostomatol 1996;193:39-57.

10. Korbendau JM,

Guyomard F.

Chirurgie muco-gingivale chez l'enfant et I'adolescent. Paris : CdP, 1991

11. Landais $\mathrm{H}$.

Germectomies précoces des dysharmonies dento-maxillaires (DDM) Rev Stomatol 1976;77(2):431-432.

12. Lerondeau JC, Schefer $P$, Verdier M. Indication des germectomies de prémolaires. Rev Stomatol 1976;77(2):441-443.

13. Maynard JG. Logique d'une thérapeutique muco-gingivale chez l'enfant et l'adolescent. Int J Periodontics Restorative Dent 1987;1:37-51.

14. Merle-Béral J, Bouhours G. Germectomie des premières prémolaires. Quand ? Comment ? Orthod Fr 1974;45(2): 203-211.

15. Prichard J. Effets sur le parodonte des extractions des prémolaires. Rev Orthop Dento Faciale 1976;10:295-296.

16. Reychler A. La prévention des germectomies. Rev Stomatol 1976:2: 444-446.

17. Sentilhes $C$.

Germectomies des prémolaires, techniques chirurgicales. Rev Odonto Stomatol 1978;7(4):307-311.

18. Van Der Linden FPGM, Duterloo HS.

Development of the human dentition. An atlas magerstown. Md. Harper \& Row eds, 1976.

19. Wilson TG, Glover ME, Schoen J, Baus C, Jacobs T. Compliance with maintenance therapy in a private periodontal practice. J Periodontol 1984;55: 468-473.

\section{SUMMARY}

\section{Transalveolar tooth germs of the first premolar teeth}

\section{G. POIROT, T. GUÉRIN}

After having glanced through various technics of premolar tooth germ removal, the authors describe a protocol of which originality is exclusively a

transalveolar approach, the advantages and drawbacks per, post operatively of these various technics.

keywords: tooth germ, surgery, bicuspid, methods. 